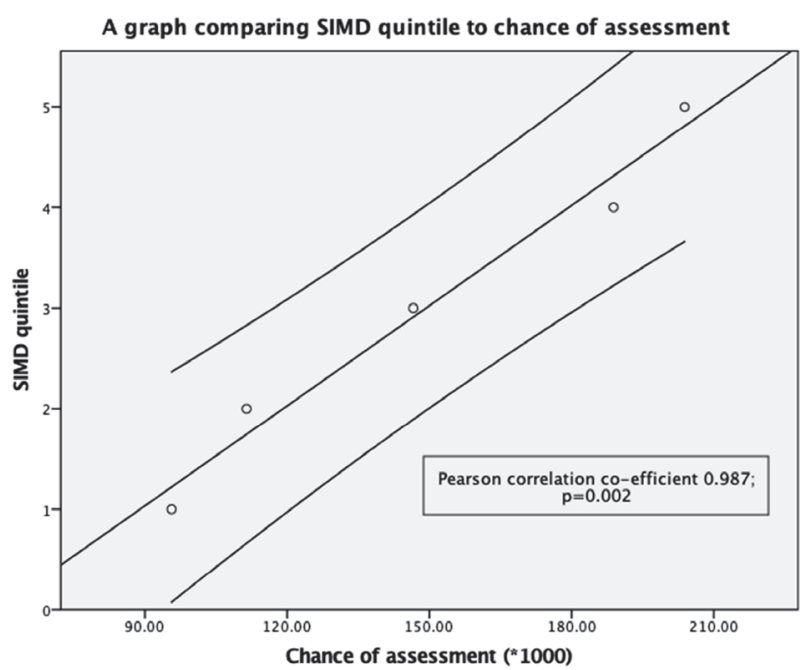

Abstract P080 Figure 1 A graph comparing SIMD quintile to chance of assessment

referrals-deaths on the list) + liver disease mortality in Scotland)

Results 1297 patients were identified. Patients from the least deprived areas of Scotland were significantly more likely to be referred for liver transplantation. Pearson Correlation co-efficient $0.987 ; p=0.002$. (See figure 1 ).

Those patients from the most deprived quintile were significantly less likely to be listed compared to the most affluent quintile. $(\mathrm{OR}=0.653 ; 95 \%$ CI $0.456-0.935 ; \mathrm{p}=0.02)$. No significant differences were found when comparing the other quintiles to the least deprived group. We did find that those from the more affluent groups did have more severe liver disease by UKELD (ANOVA $p=0.003$ ) however there was no difference in those not listed due to being 'too unwell' across the groups (Chi-squared $\mathrm{p}=0.447$ ). Number of hepatocellular carcinomas (HCC) were not significantly different across the groups (Chi-squared $\mathrm{p}=0.976$ )

Those not listed due to high risk or recidivism (Chisquared $\mathrm{p}=0.066$ ) and due to HCC outside criteria (Chisquared $\mathrm{p}=0.870$ ) were similar across the SIMD groups.

There was no difference in waiting times on the transplant list across the SIMD groups (ANOVA $\mathrm{p}=0.794$ )

We found that in those patients transplanted $(n=623)$ there was no significant difference in survival at two years when compared to the most affluent quintile. For example, when comparing the most deprived quintile (14 deaths at 2 years $(26.9 \%)$ vs 6 (11.5\%); OR $=0.600 ; 95 \%$ CI $0.222-1.621$; $\mathrm{p}=0.314)$.

Discussion Those patients from more deprived areas of Scotland are less likely to be referred or listed for liver transplantation compared to most affluent groups. However, these differences don't continue when looking at survival posttransplant.

\section{P081 DERANGED LIVER FUNCTION TESTS IN COVID POSITIVE PATIENTS REQUIRING HOSPITAL ADMISSION AT IPSWICH HOSPITAL, UK}

Usama Aslam*, Mehreen Mudassar, Mohsin Munir, Zaffar Ullah. Ipswich Hospital/esneft, Ipswich, UK

10.1136/gutjnl-2021-BASL.89
Introduction SARS-CoV-2 is a novel coronavirus that emerged in Wuhan, China in late 2019 and since become a global pandemic. Initial reports suggested a significant proportion of patients have abnormal liver blood tests. We conducted a retrospective review of clinical data to access for incidence, clinical pattern, and severity of liver blood test in patients with confirmed COVID-19.

Method Clinical records and laboratory results were obtained from 210 patients with laboratory-confirmed COVID-19 who were admitted to Ipswich Hospital, East of England, UK between March 2020 and June 2020. Only admission blood tests were reviewed and all patients who had abnormal admission liver function tests were then screened for any risk factors for liver disease. To describe the severity of liver injury, in this study, patients who had raised liver function parameters more than $5 \times$ the upper limit unit of normal (ULN) were classified as significant liver injury; patients who had raised liver function parameters 2-5 ULN were classified as moderate liver injury; and patients who had raised liver function parameters 1-2 ULN were classified as mild liver injury

Results 210 hospitalised COVID-19 positive had liver blood tests available for analysis. 44 patients (20\%) had one or more abnormal liver blood tests with raised levels of Creactive protein and procalcitonin. Abnormality in liver function test was more hepatocellular but 8 patients out of 44 had raised alkaline phosphatase only along with raised GGT but normal rest of liver enzymes. Out of these 44 patients $20(9 \%)$ required ITU admission within 24 to 48 hours of hospital admission. 15 (7\%) patients out of 44 were not ITU candidates either due to advanced age and frailty or multiple co-morbidities. 9 patients out 44 were managed on ward as were not too unwell to require ITU admission. All patient reviewed in our data had mild to moderate liver injury with none having severe injury. Factors identified in patient requiring ITU admission was male gender, age greater than 60 years and multiple comorbidities.

Conclusion Twenty percent of patients admitted to the hospital with SARS-CoV-2 infection had an abnormal liver function which was found to be associated with raised levels of inflammatory markers. Its important to aggressively treat patients with COVID-19 infection and deranged LFTS as there is a risk involved that these patients might require ITU. Its important to perform liver screen blood tests along with imaging of liver

\section{P082 VALIDATION OF THE BAVENO VI CRITERIA: IDENTIFYING LOW RISK CIRRHOTIC PATIENTS NOT REQUIRING ENDOSCOPIC SURVEILLANCE IN A TEACHING HOSPITAL}

Daniyal Baig*, John Ross Briggs, Cyril Sieberhagen. Aintree University Hospitals NHS Foundation Trust, UK

\subsection{6/gutjnl-2021-BASL.90}

Introduction In cirrhotic patients, oesophageal varices are one of the major causes of death (6-week mortality is between $17 \%-28 \%)$ and $5-11 \%$ of all cases of upper gastrointestinal bleeding (UGIB).

Patients with cirrhosis undergo variceal surveillance with oesophago-gastroduodenoscopy (OGD) to reduce the risk of oesophageal variceal bleeding. Patients with compensated 
cirrhosis will undergo endoscopic variceal surveillance every 1-3 years depending on the presence of varices.

OGD is a key diagnostic test with one of the longest waiting lists in the NHS and variceal surveillance contributes to the overall waits in upper GI endoscopy. It is therefore essential that the appropriate patients are captured in order to maximise both the efficacy of the service and also the financial cost of delivering the service.

The Baveno VI criterion uses biochemical and non-invasive measurements to predict the presence of oesophageal varices in patients with compensated cirrhosis. Based on the criteria, a platelet count $>150 \times 109 / \mathrm{L}$ and a Liver Stiffness Measurement (LSM) $<20 \mathrm{kPa}$ are deemed at low risk of oesophageal varices and could avoid an OGD for variceal surveillance.

We proposed to apply the Baveno VI criteria in our regional liver unit to identify patients that may be able to avoid having an OGD for variceal surveillance.

Methodology A retrospective analysis was completed utilising the database of cirrhotic patients registered at Aintree University Hospital, Liverpool. Patients that met Baveno VI criteria and had an OGD were included.

OGD reports from 6 July 2001 - 16 February 2021 were obtained from the endoscopy database (UniSoft). Fibroscan reports were available for patients from 26 August 2015 - 16 February 2021.

Results 623 patients were registered on the cirrhosis database of which $62(10 \%)$ fulfilled inclusion criteria. Alcohol and NASH were the predominant aetiologies $(24 \%$ and $43.5 \%$ respectively). Median age at time of Fibroscan was 60 years (30-80), median LSM was $13 \mathrm{kPa}(3.2-19.8)$, median platelet count 219x109/L (157x109/L-432x109/L). $3.2 \%$ ( 2 out of 62 ) patients had oesophageal varices (1 with Grade 1 and 1 with Grade 2 varices). 30 patients had an OGD within a year of their Fibroscan. The positive predictive value was $96.8 \%$. Varices requiring treatment in this cohort was $1.6 \%$.

Conclusion The application of Baveno VI criteria can safely and accurately decrease the number of OGD's required within a variceal surveillance program, reducing the risk of invasive procedures and resulting in a cost saving and reduction on pressures on waiting lists for OGD.

\section{P083 IMPACT OF COVID INFECTION ON OUTCOME OF PATIENTS WITH ACUTE ON CHRONIC LIVER FAILURE}

Shemin Kovammal ${ }^{*}$, Jayshri Shah, Glenda Osuoha, Seema Zulfikar, Syed Asfandyar. William Harvey Hospital, EKHUFT, Ashford, UK, Introduction

\subsection{6/gutjnl-2021-BASL.91}

Acute on chronic liver failure (ACLF) is a syndrome occurring in patients with chronic liver disease with or without cirrhosis characterised by acute hepatic decompensation and one or more extra hepatic organ failure with an increased mortality. Numerous factors have been studied which influence the outcome of these patients.

Aim To assess the impact of COVID infection on management and treatment escalation of patients who presented with acute hepatic decompensation with known or unknown underlying chronic liver disease after risk stratification.

Methodology Retrospective analysis of all patients admitted over two periods - Pre Covid (August 2019- March 2020-
Group A) and Covid (April 2020 - December 2020- Group B) with acute decompensated liver disease at our hospital. Data was analyzed from patient notes and electronic records on the following variables: age, gender, etiology of liver disease, prognostication scores - Child Pugh, MELD, ACLF grade and WHO performance status. CLIF -C score was calculated for patients referred to intensive care unit. Patient outcome measures - mortality and duration of hospital stay was also recorded. Data was collected in $35 / 72$ patients who were admitted in Group A and 31/95 patients in Group B due to non-availability of all patient notes.

Results 6/35 patients (17\%) died in Group A compared to 13/ 31(41.9\%) patients in Group B. 8/31 had COVID pneumonitis, 7 died and 1 survived. Alcohol was the commonest cause of underlying liver disease in both groups (Group A 32/35, Group B 27/31). Decompensation episodes in Group A versus Group B are as follows: gastrointestinal bleed 17/35 (48\%), 16/31(51\%); Hepatic encephalopathy 9/35(25\%), 6/31(19\%); Spontaneous Bacterial peritonitis 9/35(25\%), 9/31(29\%) respectively. Table 1 shows median MELD, Childs Pugh, ACLF grade was almost similar in both groups along with further

\section{Abstract P083 Table 1}

\begin{tabular}{|c|c|c|}
\hline & Pre Covid/Group A ( $n=35)$ & Covid/Group B $(n=31)$ \\
\hline Mean Age & $56.63 \pm 12.5$ SD & $59.16 \pm 10.75$ SD \\
\hline \multirow[t]{2}{*}{ Gender } & $21(60 \%)$ & $20(64.5 \%)$ \\
\hline & $14(40 \%)$ & $11(35.5 \%)$ \\
\hline Median MELD Score & 21(Range 6-40) & 21(Range 9-32) \\
\hline Median Child Pugh Score & 11 (Rang 6-14) & 12(Range 10-15) \\
\hline Median ACLF Score & 8 (Range 6-11) & 8 (Range $6-15$ ) \\
\hline No ACLF & $27(77 \%)$ & $21(67 \%)$ \\
\hline ACLF grade 1 & 5 & 4 \\
\hline ACLF grade 2 & 2 & 5 \\
\hline ACLF grade3 & 1 & 1 \\
\hline Average Performance score & 1 (Range $0-2$ ) & 1 (Range $0-3$ ) \\
\hline \multicolumn{3}{|l|}{ Patients referred to ITU } \\
\hline Patients referred to ITU & $4(11 \%)$ & $8(25 \%)$ \\
\hline Patients accepted for ITU & 3 & 6 \\
\hline Median CLIF - C Score & 54 (Range 45-58) & 56 (Range 51-61) \\
\hline Average Performance Score & 1 (Range $0-1$ ) & 1 (Range $0-1$ ) \\
\hline Average ITU days & 9 (Range $6-13$ ) & 7 (Range 1-16) \\
\hline Mortality & $1(25 \%)$ & $4(66.6 \%)$ \\
\hline \multicolumn{3}{|c|}{ Patients who received ward-based care } \\
\hline $\begin{array}{l}\text { Number of patients admitted for } \\
\text { ward-based care }\end{array}$ & $32(91.4 \%)$ & $25(80.6 \%)$ \\
\hline Median MELD score & 18 (Range 6-27) & 21 (Range (9-32) \\
\hline Average hospital days & 14 days (Range $3-80$ ) & 16 days (Range $3-31$ ) \\
\hline Mortality & $5(16 \%)$ & $9(36 \%)$ \\
\hline $\begin{array}{l}\text { Median MELD score for patients } \\
\text { with mortality }\end{array}$ & 21 (Range 21- 28) & 26 (Range 13- 40) \\
\hline \multicolumn{3}{|c|}{ Patients who recovered \& got Discharged } \\
\hline Number of patients & $29(82.8 \%)$ & $18(58 \%)$ \\
\hline Median MELD score & 15 (Range 6-22) & 19 (Range 9 - 28) \\
\hline Median ACLF score & 7(Range 6- 11) & 8(Range 6- 11) \\
\hline Average Performance Score & 1 (Range 0 - 2) & 1 (Range $0-2$ ) \\
\hline \multicolumn{3}{|l|}{ Patients who Died } \\
\hline Number of patients & $6(17 \%)$ & $13(41 \%)$ \\
\hline Median MELD Score & 21(Range $18-28$ ) & 28 (Range 13-40) \\
\hline Median ACLF score & 8 (Range 6-11) & 9 (Range 6 -15) \\
\hline Average Performance Score & 1 (Range $0-2$ ) & 1(Range $0-2$ ) \\
\hline
\end{tabular}

